\title{
Establishing a regular systems-based teaching programme for core medical trainees within a district general hospital
}

\author{
Authors: Kate Edwards* and Tom Cozens
}

\section{Introduction}

Although Health Education and Improvement Wales (HEIW) provide regional teaching days for core medical trainees (CMTs) in line with the curriculum, the local teaching for CMTs within hospitals in Aneurin Bevan University Health Board has been variable. Focusing on the Royal Gwent Hospital where no formal teaching was being run locally, I aimed to establish regular teaching sessions for CMTs incorporating clinical skills, simulation training and useful systems-based discussions that are relevant to day to day working practices.

The aim is to drive cultural change in a hospital that has never run local teaching sessions for CMTs to establish a regular programme that can be continued beyond August 2019 with the new introduction of internal medicine trainees in Wales.

\section{Materials and methods}

Having initially asked the current cohort of CMTs what type of teaching they would like to receive, it was apparent that most CMTs wished to have clinical skills and simulation teaching to help them gain confidence in core procedures and emergency scenarios as they progress into higher training.

With this in mind I set up discussions with the simulation lead for the hospital and the postgraduate department to organise dates and times that these sessions could be run. As a gastroenterology trainee, I set up a month in November 2018 where I hosted teaching for the CMTs on a weekly basis to include a clinical skills station on ascitic drain and Sengstaken-Blakemore tube insertion, a simulation session on management on gastrointestinal (GI) bleeds and decompensated liver disease, and small group talks on the initial management of luminal and hepatology emergencies that are commonly encountered on the medical intake.

The feedback received from these four sessions was very positive with many CMTs asking for continued regular teaching using this format. I subsequently utilised this feedback to gain support at the medical directorate meeting in creating a medical simulation faculty to ensure longevity of this teaching programme.

Since January 2019, a regular teaching programme delivered once a week has been established, with each month focusing on

Authors: Aneurin Bevan University Health Board *RCP chief registrar one medical specialty, which to date has included cardiology and acute medicine. Feedback from these teaching sessions has again been very positive and is helping to drive change over the next 6 months.

\section{Results and discussion}

To date, the teaching sessions have been rated overall by attendees as 'excellent' or 'very good'. Two-thirds of people felt the clinical skills workshops in particular improved their clinical skills 'a great amount' and all attendees agreed that these workshops would help them pass their Annual Review of Competency Progression (ARCP) for their level of training.

Having established clinical skills sessions in previous hospitals I was somewhat confident that I had the understanding to set up this teaching programme locally. However, I have experienced some challenges along the way. Skills gained so far in my chief registrar training have helped a great deal in overcoming these challenges. The challenges included agreeing on a set day for the regular teaching sessions as many medical teams hold departmental teaching or multi-disciplinary team meetings at lunchtime. Negotiations with different clinical directors regarding the day and time of CMT teaching led to agreement on a Thursday lunchtime prior to care of the elderly departmental teaching. After running the first few teaching sessions on a Thursday lunchtime, attendance was relatively poor despite good communication beforehand with the CMTs. Feedback suggested that CMTs felt the postgraduate department, being detached from the main hospital building, was one factor that contributed to poor attendance. In response to this, I moved any non-clinical based teaching sessions to the medical mess which is central to the hospital and has facilities to hold meetings and run PowerPoint presentations. With this change, uptake was much improved, however it still look a lot of messages via the CMT WhatsApp group and emails to remind juniors that this teaching is now a permanent and a regular feature of Thursday lunchtimes.

Finally, to ensure longevity of this teaching programme, I have approached the medical directorate to aid the establishment of a medical faculty for simulation, something that is mandatory for internal medicine trainees from August in Wales. Uptake from consultants has been slow, with many expressing concerns that simulation is not a modality of teaching they are used to doing and do not have the skill set to teach. As a result, 'Train the trainers' sessions are being run locally in the next few months to equip medical consultants with the skills needed to run simulation scenarios, including debriefing. 


\section{Conclusion}

Establishing a regular local teaching programme for core medical trainees has had its challenges, particularly driving a culture towards positive change among trainees and consultant colleagues in a hospital which has traditionally had no formal teaching.
Despite the challenges faced, the outcome of establishing this varied systems-based teaching programme has proved to be beneficial to trainees to date, helping them to gain confidence in both academic knowledge and clinical skills which can be utilised as they progress into higher training posts. 